\title{
All-Cause Mortality and Serious Cardiovascular Events in People with Hip and Knee Osteoarthritis: A Population Based Cohort Study
}

\author{
Gillian A. Hawker ${ }^{1,2,3,4_{*}}$, Ruth Croxford ${ }^{3}$, Arlene S. Bierman ${ }^{2,3,4,5}$, Paula J. Harvey ${ }^{1,4}$, Bheeshma Ravi ${ }^{2,6}$, \\ lan Stanaitis ${ }^{1}$, Lorraine L. Lipscombe ${ }^{1,2,3,4}$
}

1 Women's College Research Institute, Women's College Hospital, Toronto, Ontario, Canada, $\mathbf{2}$ Institute of Health Policy, Management and Evaluation, University of Toronto, Toronto, Ontario, Canada, 3 Institute for Clinical Evaluative Sciences, Toronto, Ontario, Canada, 4 Department of Medicine, University of Toronto, Toronto, Ontario, Canada, 5 Lawrence S. Bloomberg Faculty of Nursing, University of Toronto, Toronto, Ontario, Canada, 6 Division of Orthopaedics, Department of Surgery, University of Toronto, Toronto, Ontario, Canada

\begin{abstract}
Background: Because individuals with osteoarthritis (OA) avoid physical activities that exacerbate symptoms, potentially increasing risk for cardiovascular disease (CVD) and death, we assessed the relationship between OA disability and these outcomes.

Methods: In a population cohort aged 55+ years with at least moderately severe symptomatic hip and/or knee OA, OA disability (Western Ontario McMaster Universities (WOMAC) OA scores; Health Assessment Questionnaire (HAQ) walking score; use of walking aids) and other covariates were assessed by questionnaire. Survey data were linked to health administrative data to determine the relationship between baseline OA symptom severity to all-cause mortality and occurrence of a composite CVD outcome (acute myocardial infarction, coronary revascularization, heart failure, stroke or transient ischemic attack) over a median follow-up of 13.2 and 9.2 years, respectively.
\end{abstract}

Results: Of 2156 participants, 1,236 (57.3\%) died and 822 (38.1\%) experienced a CVD outcome during follow-up. Higher (worse) baseline WOMAC function scores and walking disability were independently associated with a higher all-cause mortality (adjusted hazard ratio, aHR, per 10-point increase in WOMAC function score 1.04, 95\% confidence interval, $\mathrm{Cl} 1.01-$ $1.07, p=0.004$; aHR per unit increase in HAQ walking score $1.30,95 \% \mathrm{Cl} 1.22-1.39, \mathrm{p}<0.001$; and aHR for those using versus not using a walking aid $1.51,95 \% \mathrm{Cl} 1.34-1.70, \mathrm{p}<0.001)$. In survival analysis, censoring on death, risk of our composite CVD outcome was also significantly and independently associated with greater baseline walking disability ((aHR for use of a walking aid $=1.27,95 \% \mathrm{Cl} 1.10-1.47, \mathrm{p}=0.001$; a HR per unit increase in $\mathrm{HAQ}$ walking score $=1.17,95 \% \mathrm{Cl} 1.08-1.27, \mathrm{p}<$ 0.001 ).

Conclusions: Among individuals with hip and/or knee OA, severity of OA disability was associated with a significant increase in all-cause mortality and serious CVD events after controlling for multiple confounders. Research is needed to elucidate modifiable mechanisms.

Citation: Hawker GA, Croxford R, Bierman AS, Harvey PJ, Ravi B, et al. (2014) All-Cause Mortality and Serious Cardiovascular Events in People with Hip and Knee Osteoarthritis: A Population Based Cohort Study. PLoS ONE 9(3): e91286. doi:10.1371/journal.pone.0091286

Editor: Robert B. Sim, Oxford University, United Kingdom

Received August 14, 2013; Accepted February 11, 2014; Published March 7, 2014

Copyright: (C) 2014 Hawker et al. This is an open-access article distributed under the terms of the Creative Commons Attribution License, which permits unrestricted use, distribution, and reproduction in any medium, provided the original author and source are credited.

Funding: This study was funded by an operating grant from the Canadian Institutes of Health Research, grant \# MOP-15468. This study was also supported by the Institute for Clinical Evaluative Sciences (ICES), which is funded by an annual grant from the Ontario Ministry of Health and Long-Term Care. The funders had no role in study design, data collection and analysis, decision to publish, or preparation of the manuscript. No endorsement by ICES or the Ontario Ministry of Health and Long-Term Care is intended or should be inferred. ICES had no role in the study design, collection, analysis, or interpretation of the data, writing of the report, or in the decision to submit the report for publication.

Competing Interests: We have read the journal's policy and have the following conflicts: Gillian Hawker was supported by a salary award from The Arthritis Society of Canada and holds the FM Hill Chair in Academic Women's Medicine. Lorraine Lipscombe is supported by a CIHR New Investigator Award. The other authors report no competing interests. This does not alter our adherence to PLOS ONE policies on sharing data and materials.

* E-mail: g.hawker@utoronto.ca

\section{Introduction}

Globally, aging populations and the growing prevalence of obesity $[1,2]$ have led to increased population risk for hypertension [3,4], dyslipidemia [5], diabetes [6], and cardiovascular disease (CVD) [7]. A less well recognized consequence of these trends is the increasing burden of osteoarthritis $(\mathrm{OA})[5,8]$. OA is the most common arthritis [9], affecting approximately $10 \%$ of the adult population [1]. Symptomatic OA leads to functional limitations, depressed mood and loss of independence [10]. With no cure, OA management focuses on pain relief and preserving physical function [11] using non-pharmacologic and pharmacologic therapies and, ultimately, joint replacement surgery. Despite effective management strategies, OA is under-diagnosed and under-treated. This is in part due to the high co-prevalence of other chronic conditions in people with OA; $90 \%$ are estimated to 
have at least one additional chronic condition [12], with OA and CVD among the most common dyads seen in clinical practice. CVD, in particular, may be perceived as precluding the use of OA therapies (e.g. non-steroidal anti-inflammatory drugs, NSAIDs $[13,14])$.

Inadequately treated, people commonly manage OA pain by avoiding activities, like walking, that exacerbate pain [10,15]. This lack of physical activity may in turn lead to poorer fitness, higher risk of CVD [16,17], and inadequate self-management of chronic conditions [18]. Nuesch et al [19] documented increased all-cause mortality in people with versus without OA in an English population cohort; walking disability predicted increased risk for death. Given the rising numbers with OA, confirmation of these relationships is important. Further, it is important to know whether among people with hip/knee OA, severity of OA-related functional limitations predicts adverse outcomes, including serious CVD events and death. Documenting a significant, independent relationship between OA symptom severity and CVD risk would provide evidence for improving OA management to reduce CVD risk and for screening and modifying CVD risk factors in people with OA. In a Canadian population cohort with hip and knee OA, the current study therefore evaluated the prognostic value of baseline levels of hip and knee osteoarthritis $(\mathrm{OA})$ related pain and disability on all-cause mortality and risk for serious GVD events.

\section{Methods}

\section{Ethics Statement}

Participants provided written informed consent to participate in the study. The Women's College Research Institute Ethics Review Board approved the study.

\section{Participants}

The Ontario Hip and Knee Study is a population-based study of people who reported at least moderately severe pain and disability from hip and/or knee OA. The cohort was recruited from 1996-98 through a screening survey of $100 \%$ of the population aged 55+ years in two Ontario regions - one rural and one urban - that identified individuals with symptomatic hip/ knee arthritis [20]. Of 28,451 screening survey respondents $(72.3 \%$ response rate), those with: difficulty in the last three months with each of stair climbing, rising from a chair, standing and walking; swelling, pain or stiffness in any joint lasting at least six weeks; and who indicated on a diagram that a hip or knee had been 'troublesome' were invited to participate. Of 2,411 that agreed, 2,225 had OA (Figure 1). A subsequent validation study found that $96 \%$ who met cohort criteria had hip and/or knee arthritis on examination and radiographs.

\section{Assessments}

We used data collected at baseline through a standardized mail/ telephone survey linked, with participants' consent, to provincial health administrative databases: a) physician services from the Ontario Health Insurance Plan (OHIP) Physician and Laboratory Billing Records; b) inpatient hospitalizations and same day surgeries from the Canadian Institute for Health Information (CIHI) hospital discharge abstract database (DAD) and National Ambulatory Care Reporting System (NACRS) database (from April, 2002); c) Emergency Department (ED) visits - prior to April, 2002, extracted from the Physician Billing Records; as of April, 2002, ED visits were obtained from the NACRS database; and prescription drug use from the Ontario Drug Benefits records, for those aged $65+$ years and thus eligible for provincial drug benefits.
The baseline survey assessed: socio-demographics; living circumstances; smoking; self-reported height, weight and NSAID use; number of troublesome (painful, aching, stiff or swollen) hips and knees using a joint homunculus; comorbidity (participants indicated if they had been diagnosed by a physician and received treatment in the past year for 'high blood pressure', 'hardening of the arteries', diabetes, and 'heart problems'); and mental health status (SF-36 mental health subscale; scores $<60 / 100$ indicate probable depression [21]). OA symptom severity was assessed using the reliable and valid Western Ontario McMaster Universities OA Index, WOMAC, pain and physical function subscales [22]. The five-item pain subscale assesses the amount of pain experienced in the hips and knees in the previous week with walking on flat ground, going up/down stairs, at night in bed, sitting or lying, and standing upright. The 17-item physical function subscale assesses the amount of difficulty experienced in the past week performing each of 17 activities ranging from sitting to heavy household chores. Response options are from 0 (none) to 4 (extreme). For each scale, item scores were summed and then normalized to lie between 0 and 100; higher scores indicate worse OA symptoms. Baseline walking disability was additionally assessed using the 2-item Health Assessment Questionnaire [23], HAQ, walking subscale, and use of an aid (e.g., cane or walker) for ambulation (yes/no). The HAQ walking scale assesses level of difficulty in the prior week walking outdoors on flat ground and climbing up five steps, from none (0) to unable to do (3), and use of aids or devices to perform these activities. Higher scores indicate greater difficulty walking.

The presence of hypertension or diabetes at baseline was based on either self-reported physician diagnosis 'ever' or meeting validated criteria for inclusion in provincial disease registries [24,25]. History of CVD prior to cohort recruitment (pre-existing CVD) was defined as 'present' if the participant had experienced $\geq 1$ hospital discharge for acute myocardial infarction (AMI), congestive heart failure (CHF), stroke or transient ischemic attack (TIA), or receipt of coronary revascularization, within the previous five years [26]. Using physician claims, the number of ambulatory visits to a primary care physician and specialists in the pre-baseline year were assessed as proxies for health status. The Johns Hopkins University Adjusted Clinical Group system was used to classify participants as 'frail' (yes/no) [27]. Receipt of post-baseline hip or knee joint replacement procedures (elective and non-elective primary and revision TJA) was determined using hospital discharge data; the procedure and diagnostic codes used to identify joint replacement procedures may be found at http:// links.lww.com/A1274.

Our primary outcome was all cause-mortality from baseline to February 28, 2012, determined from hospital discharge abstracts and death certificates from the Registered Persons Database. Our secondary outcome was a composite CVD outcome, defined as an $\mathrm{ED}$ visit or hospitalization for $\mathrm{AMI}$, coronary revascularization (CABG or PCI), CHF, stroke or TIA.

\section{Statistical Analysis}

Baseline cohort characteristics and the proportions that experienced each outcome were calculated using proportions, means and medians as appropriate. Cox proportional hazards regression was used to examine the contribution of baseline $\mathrm{OA}$ symptom severity (WOMAC pain subscale, WOMAC function subscale, HAQ walking score and use of a walking aid, separately) to all-cause mortality after controlling for potential confounders and to examine time to our composite CVD outcome, censoring on death. For each model, we assessed the following baseline covariates: socio-demographics, probable depression, hyperten- 


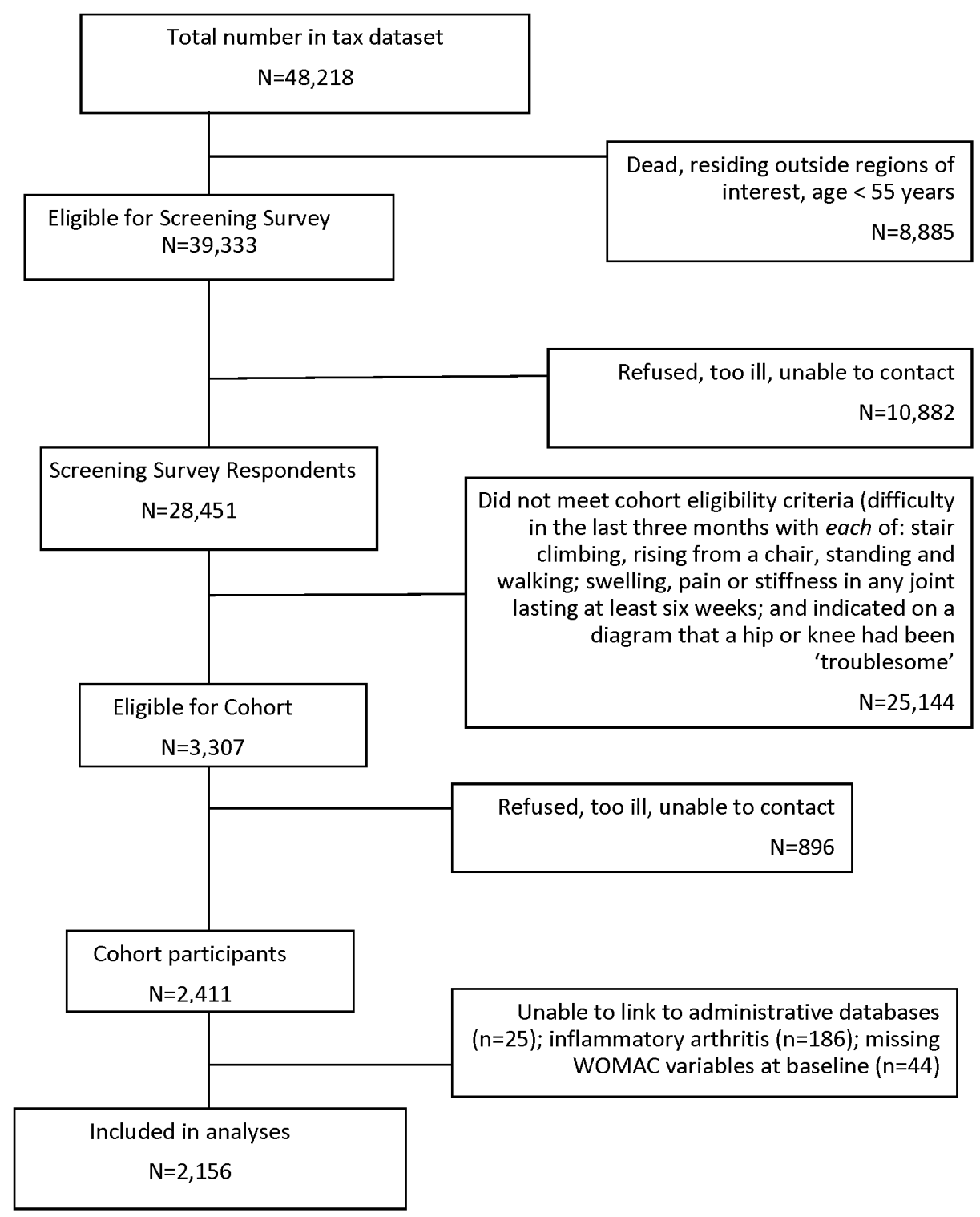

Figure 1. Cohort recruitment flow chart.

doi:10.1371/journal.pone.0091286.g001

sion, diabetes, pre-existing CVD, number of other self-reported conditions, body mass index (BMI), smoking, and self-reported NSAID use. Missing baseline values for height, weight or smoking status were imputed using post-baseline information. Age, sex, BMI, smoking status, diabetes, pre-existing CVD, and hypertension were included in all multivariable models; additional covariates were those associated with the outcome of interest at $\mathrm{p}<0.05$. In our final models, we tested for interactions between baseline OA severity (WOMAC scores, HAQ walking score, use of walking aids) and pre-existing CVD.

Secondary analyses. Non-ASA NSAIDs have been shown to increase risk for CVD events. Thus, the relationship between worse OA symptoms and occurrence of CVD events or death, if found, may be explained, in part, by use of prescription NSAIDs. In a secondary analysis, we therefore controlled for receipt of a prescription for a non-ASA NSAID in cohort members eligible for drug benefits during the pre-baseline year (age 66+ years at baseline). Finally, we have previously shown that, in the absence of receipt of total joint arthroplasty (TJA), people with hip and knee OA experience worsening of their functional limitations over time $[28,29]$; receipt of primary, elective TJA of the hip or knee is associated with significant improvement in OA pain and walking disability. Thus, we also assessed the effect of baseline measures of OA disability and our outcomes of interest after further adjustment for post-baseline receipt of a primary, elective hip or knee TJA, modeled as a time-dependent covariate. We hypothesized that receipt of TJA would be associated with an attenuation of the effect of baseline OA severity on our primary and secondary outcomes.

In all analyses, individuals were censored if they emigrated, died or at the end of available data (February 28, 2012). Analyses were conducted using SAS (Version 9, SAS Institute, Cary, North Carolina). 
Table 1. Sample characteristics by baseline level of physical function (WOMAC Function Subscale Quartiles).

\begin{tabular}{|c|c|c|c|c|c|c|}
\hline \multirow[b]{2}{*}{ Participant Characteristic } & \multirow[b]{2}{*}{$\begin{array}{l}\text { Overall } \\
(\mathrm{N}=2156)\end{array}$} & \multicolumn{4}{|c|}{ WOMAC Function Quartile } & \multirow[b]{2}{*}{ P-value* } \\
\hline & & $\begin{array}{l}1^{\text {st }} \text { (least } \\
\text { affected) } \\
(\mathrm{N}=502)\end{array}$ & $2^{n d}(N=530)$ & $3^{\text {rd }}(N=557)$ & $\begin{array}{l}4^{\text {th }} \text { (most } \\
\text { affected) } \\
(\mathrm{N}=567)\end{array}$ & \\
\hline \multicolumn{7}{|l|}{$\begin{array}{l}\text { Demographics } \\
\text { (Baseline Assessment) }\end{array}$} \\
\hline Age: mean (SD) & $71.3(9.2)$ & $70.4(8.9)$ & $70.4(8.7)$ & $72.0(9.0)$ & $72.1(10.1)$ & $<0.001$ \\
\hline Age category: N (\%) & & & & & & $<0.001$ \\
\hline $55-64$ & $591(27.4 \%)$ & $150(29.9 \%)$ & $148(27.9 \%)$ & $133(23.9 \%)$ & $160(28.2 \%)$ & \\
\hline 65-74 & 767 (35.6\%) & $192(38.3 \%)$ & $220(41.5 \%)$ & $194(34.8 \%)$ & $161(28.4 \%)$ & \\
\hline $75+$ & $798(37.0 \%)$ & $160(31.9 \%)$ & $162(30.6 \%)$ & $230(41.3 \%)$ & $246(43.4 \%)$ & \\
\hline Sex: N (\%) female & $1,552(72.0 \%)$ & $348(69.3 \%)$ & $366(69.1 \%)$ & $401(72.0 \%)$ & $437(77.1 \%)$ & 0.01 \\
\hline Income: N (\%) & & & & & & $<0.001$ \\
\hline$\leq \$ 20,000$ & $1,132(52.5 \%)$ & $220(43.8 \%)$ & $251(47.4 \%)$ & $293(52.6 \%)$ & $368(64.9 \%)$ & \\
\hline$>\$ 20,000$ & $649(30.1 \%)$ & $198(39.4 \%)$ & $187(35.3 \%)$ & $163(29.3 \%)$ & $101(17.8 \%)$ & \\
\hline Missing & $375(17.4 \%)$ & $84(16.7 \%)$ & $92(17.4 \%)$ & $101(18.1 \%)$ & $98(17.3 \%$ & \\
\hline Education: N (\%) & & & & & & $<0.001$ \\
\hline Less than high school completion & $778(36.1 \%)$ & $145(28.9 \%)$ & $161(30.4 \%)$ & $221(39.7 \%)$ & $251(44.3 \%)$ & \\
\hline Completed high school & $1,013(47.0 \%)$ & $247(49.2 \%)$ & $263(49.6 \%)$ & $266(47.8 \%)$ & $237(41.8 \%)$ & \\
\hline Some post-secondary education & $327(15.2 \%)$ & $105(20.9 \%)$ & $100(18.9 \%)$ & $60(10.8 \%)$ & $62(10.9 \%)$ & \\
\hline Missing & $38(1.8 \%)$ & $5(1.0 \%)$ & $6(1.1 \%)$ & $10(1.8 \%)$ & $17(3.0 \%)$ & \\
\hline Living arrangements: $\mathrm{N}(\%)$ & & & & & & 0.02 \\
\hline Living with others & $1,425(66.1 \%)$ & $350(69.7 \%)$ & $374(70.6 \%)$ & $360(64.6 \%)$ & $341(60.1 \%)$ & \\
\hline Living alone & $671(31.1 \%)$ & $138(27.5 \%)$ & $145(27.4 \%)$ & $183(32.9 \%)$ & $205(36.2 \%)$ & \\
\hline In long-term care & $33(1.5 \%)$ & $9(1.8 \%)$ & $5(0.9 \%)$ & $7(1.3 \%)$ & $12(2.1 \%)$ & \\
\hline Missing & $27(1.3 \%)$ & $5(1.0 \%)$ & $6(1.1 \%)$ & $7(1.3 \%)$ & $9(1.6 \%)$ & \\
\hline Place of residence: $\mathrm{N}(\%)$ urban & $942(43.7 \%)$ & $271(44.0 \%)$ & $225(42.5 \%)$ & $225(40.4 \%)$ & $271(47.8 \%)$ & 0.08 \\
\hline \multicolumn{7}{|l|}{$\begin{array}{l}\text { Health-Related Variables } \\
\text { (Baseline Assessment) }\end{array}$} \\
\hline $\begin{array}{l}\text { Pre-existing CVD (self-reported or } \\
\text { documented in the administrative data): N (\%) }\end{array}$ & $854(39.6 \%)$ & 159 (31.7\%) & $188(35.5 \%)$ & $233(41.8 \%)$ & $274(48.3 \%)$ & $<0.001$ \\
\hline $\begin{array}{l}\text { Diabetes (self reported or documented } \\
\text { in the administrative data): } \mathrm{N}(\%)\end{array}$ & $440(20.4 \%)$ & $87(17.3 \%)$ & $95(17.9 \%)$ & $114(20.5 \%)$ & $144(25.4 \%)$ & 0.003 \\
\hline $\begin{array}{l}\text { Hypertension (self reported or } \\
\text { documented in the administrative data): } \\
\mathrm{N}(\%)\end{array}$ & $1,369(63.5 \%)$ & $302(60.2 \%)$ & $323(60.9 \%)$ & $366(65.7 \%)$ & $378(66.7 \%)$ & 0.06 \\
\hline $\begin{array}{l}\text { Depression (SF36 mental health subscale): } \\
\mathrm{N}(\%)\end{array}$ & $621(28.8 \%)$ & $83(16.5 \%)$ & $123(23.2 \%)$ & $154(27.7 \%)$ & $261(46.0 \%)$ & $<0.001$ \\
\hline $\begin{array}{l}\text { No. other comorbid conditions (self reported): } \\
\mathrm{N}(\%)\end{array}$ & & & & & & $<0.001$ \\
\hline 0 & $84(3.9 \%)$ & $37(7.4 \%)$ & $19(3.6 \%)$ & $12(2.2 \%)$ & $16(2.8 \%)$ & \\
\hline 1 & 390 (18.1\%) & $128(25.5 \%)$ & $101(19.1 \%)$ & 105 (18.9\%) & $56(9.9 \%)$ & \\
\hline 2 & $678(31.5 \%)$ & $176(35.1 \%)$ & $178(33.6 \%)$ & $186(33.4 \%)$ & $138(24.3 \%)$ & \\
\hline $3+$ & $1,004(46.6 \%)$ & $161(32.1 \%)$ & $232(43.8 \%)$ & $254(45.6 \%)$ & $357(63.0 \%)$ & \\
\hline Frailty: N (\%) & $54(2.5 \%)$ & $12(2.4 \%)$ & $7(1.3 \%)$ & $16(2.9 \%)$ & $19(3.4 \%)$ & 0.17 \\
\hline Body mass index (BMI): N (\%) & & & & & & 0.007 \\
\hline Underweight & $36(1.7 \%)$ & $5(1.0 \%)$ & $6(1.1 \%)$ & $12(2.2 \%)$ & $13(2.3 \%)$ & \\
\hline Normal & $546(25.4 \%)$ & $152(30.3 \%)$ & $127(24.0 \%)$ & $148(26.6 \%)$ & $119(21.0 \%)$ & \\
\hline Overweight & $761(35.3 \%)$ & $181(36.1 \%)$ & $200(37.7 \%)$ & $188(33.8 \%)$ & $192(33.9 \%)$ & \\
\hline Obese & $683(31.7 \%)$ & $136(27.1 \%)$ & $174(32.8 \%)$ & $170(30.5 \%)$ & $203(35.8 \%)$ & \\
\hline Missing & $130(6.0 \%)$ & $28(5.6 \%)$ & $23(4.3 \%)$ & 39 (7.0\%) & $40(7.1 \%)$ & \\
\hline Smoking history: N (\%) & & & & & & 0.55 \\
\hline Never smoked & $1,012(46.9 \%)$ & $231(46.0 \%)$ & $239(45.1 \%)$ & $270(48.5 \%)$ & $272(48.0 \%)$ & \\
\hline
\end{tabular}


Table 1. Cont.

\begin{tabular}{|c|c|c|c|c|c|c|}
\hline \multirow[b]{2}{*}{ Participant Characteristic } & \multirow[b]{2}{*}{$\begin{array}{l}\text { Overall } \\
(N=2156)\end{array}$} & \multicolumn{4}{|c|}{ WOMAC Function Quartile } & \multirow[b]{2}{*}{ P-value } \\
\hline & & $\begin{array}{l}1^{\text {st }} \text { (least } \\
\text { affected) } \\
(\mathrm{N}=502)\end{array}$ & $2^{\text {nd }}(N=530)$ & $3^{\text {rd }}(N=557)$ & $\begin{array}{l}4^{\text {th }} \text { (most } \\
\text { affected) } \\
(\mathrm{N}=567)\end{array}$ & \\
\hline Former smoker & $783(36.3 \%)$ & 194 (38.7\%) & 197 (37.2\%) & $205(36.8 \%)$ & $187(33.0 \%)$ & \\
\hline Current smoker & $312(14.5 \%)$ & $67(13.4 \%)$ & $81(15.3 \%)$ & $72(12.9 \%)$ & $92(16.2 \%)$ & \\
\hline Missing & $49(2.3 \%)$ & $10(2.0 \%)$ & $13(2.5 \%)$ & $10(1.8 \%)$ & $16(2.8 \%)$ & \\
\hline $\begin{array}{l}\text { Number of visits to a general practitioner in } \\
\text { pre-baseline year: median (inter-quartile } \\
\text { range) }\end{array}$ & $6(3-11)$ & $6(2-10)$ & $5(3-10)$ & $6(3-11)$ & $8(4-12)$ & $<0.001$ \\
\hline $\begin{array}{l}\text { Number of visits to a specialist in pre-baseline } \\
\text { year: median (inter-quartile range) }\end{array}$ & $2(1-6)$ & $2(1-6)$ & $2(0-5)$ & $2(1-6)$ & $3(1-7)$ & 0.005 \\
\hline Continuity of Care Index (range is from 0 to 1 ) & $0.90(0.74-1.0)$ & $0.90(0.71-1.0)$ & $0.93(0.76-1.0)$ & $0.90(0.72-1.0)$ & $0.89(0.74-1.0)$ & 0.03 \\
\hline \multicolumn{7}{|l|}{ Osteoarthritis-Related Variables } \\
\hline Use of aid for walking: $\mathrm{N}(\%)$ & $953(44.2 \%)$ & $130(25.9 \%)$ & 179 (33.8\%) & $267(47.9 \%)$ & 377 (66.5\%) & $<0.001$ \\
\hline HAQ walking score (/3): median (IQR) & $2(0-2)$ & $0(0-2)$ & $1(0-2)$ & $2(1-2)$ & $2(2-2)$ & $<0.0001$ \\
\hline WOMAC pain quartile: $\mathrm{N}(\%)$ & & & & & & $<0.001$ \\
\hline $1^{\text {st }}$ (least pain) & $460(21.3 \%)$ & $317(63.2 \%)$ & $97(18.3 \%)$ & $33(5.9 \%)$ & $13(2.3 \%)$ & \\
\hline $2^{\text {nd }}$ & $435(20.2 \%)$ & $116(23.1 \%)$ & $210(39.6 \%)$ & $92(16.5 \%)$ & $17(3.0 \%)$ & \\
\hline $3^{\text {rd }}$ & $627(29.2 \%)$ & $59(11.8 \%)$ & $172(32.5 \%)$ & $262(47.0 \%)$ & $134(23.6 \%)$ & \\
\hline $4^{\text {th }}$ (most pain) & $627(29.2 \%)$ & $7(1.4 \%)$ & $49(9.3 \%)$ & $169(30.3 \%)$ & $402(70.9 \%)$ & \\
\hline Missing & $7(0.3 \%)$ & $3(0.6 \%)$ & $2(0.4 \%)$ & $1(0.2 \%)$ & $1(0.2 \%)$ & \\
\hline Non-ASA NSAID use (self-report): N (\%) & $816(37.9 \%)$ & $163(32.5 \%)$ & $222(41.9 \%)$ & $211(37.9 \%)$ & $220(38.8 \%)$ & 0.02 \\
\hline Number of troublesome hips/knees: $\mathrm{N}(\%)$ & & & & & & $<0.001$ \\
\hline 0 & $84(3.9 \%)$ & $44(8.8 \%)$ & $21(4.0 \%)$ & $9(1.6 \%)$ & $10(1.8 \%)$ & \\
\hline 1 & $310(14.4 \%)$ & $101(20.1 \%)$ & $94(17.7 \%)$ & $77(13.8 \%)$ & $38(6.7 \%)$ & \\
\hline 2 & $786(36.5 \%)$ & 179 (35.7\%) & 199 (37.6\%) & $213(38.2 \%)$ & $195(34.4 \%)$ & \\
\hline 3 & $249(11.6 \%)$ & $39(7.8 \%)$ & $59(11.1 \%)$ & $75(13.5 \%)$ & $76(13.4 \%)$ & \\
\hline 4 & $454(21.1 \%)$ & $35(7.0 \%)$ & $103(19.4 \%)$ & $109(19.6 \%)$ & 207 (36.5\%) & \\
\hline Missing & $273(12.7 \%)$ & $104(20.7 \%)$ & $54(10.2 \%)$ & $74(13.3 \%)$ & $41(7.2 \%)$ & \\
\hline \multicolumn{7}{|l|}{ Follow-up Time and Outcomes } \\
\hline $\begin{array}{l}\text { Follow-up time until death or censoring (years): } \\
\text { median (inter-quartile range) }\end{array}$ & $13.2(6.3-15.5)$ & $13.9(7.5-15.5)$ & $13.9(7.8-15.6)$ & $13.0(5.8-15.5)$ & $10.6(5.0-15.3)$ & $<0.001$ \\
\hline Outcome of death: $\mathrm{N}(\%)$ & $1,236(57.3 \%)$ & $269(21.8 \%)$ & $265(21.4 \%)$ & $331(26.8 \%)$ & $371(30.0 \%)$ & $<0.001$ \\
\hline $\begin{array}{l}\text { Follow-up time until composite CVD } \\
\text { outcome or censoring): median (inter-quartile } \\
\text { range) }\end{array}$ & $9.2(3.7-15.0)$ & $10.5(4.8-15.2)$ & $10.6(4.7-15.4)$ & $8.8(3.3-14.9)$ & $6.9(3.1-14.6)$ & $<0.001$ \\
\hline $\begin{array}{l}\text { CVD-related hospital admission or } \\
\text { ED visit: } N(\%)\end{array}$ & $822(38.1 \%)$ & $182(36.3 \%)$ & $189(35.7 \%)$ & 213 (38.2\%) & $238(42.0 \%)$ & 0.13 \\
\hline
\end{tabular}

$\mathrm{CVD}=$ Cardiovascular disease

*P-values test the hypothesis that of no differences among the four WOMAC function quartiles and were obtained using ANOVA (continuous, normally distributed covariates), Wilcoxon rank sum tests (ordinal covariates), and chi-square tests (categorical covariates).

doi:10.1371/journal.pone.0091286.t001

\section{Results}

\section{Baseline Cohort Characteristics}

Of the 2,225 baseline cohort participants with hip/knee OA, 25 could not be linked with administrative data and 44 were missing baseline WOMAC function scores, leaving 2,156 individuals for our analyses (Figure 1). Their mean age was 71.3 years (SD 9.2) and $72.0 \%$ were female; $39.6 \%$ had pre-existing CVD, $20.4 \%$ had diabetes and $63.5 \%$ hypertension, $28.8 \%$ met criteria for probable depression, $33.7 \%$ were obese and $37.2 \%$ and $14.8 \%$ were past and current smokers, respectively. Mean baseline WOMAC pain and function scores (/100) were 40.9 (SD 21.9) and 40.6 (SD 20.8), respectively. Forty-four percent reported using walking aids; $37.9 \%$ reported NSAID use. Table 1 .

\section{Effect of Baseline OA Pain and Disability on All-Cause Mortality}

Over a median 13.2 years of follow-up (inter-quartile range, IQR, 6.3-15.5 years), 1,236 (57.3\%) participants died. Measures of baseline OA-related disability, but not pain, were associated with increased risk for all cause death in unadjusted analyses (Hazard Ratio, HR, per 10-point increase in WOMAC function score 1.08, 95\% Confidence Interval, CI, 1.05-1.11, $\mathrm{p}<0.001$; HR per unit increase in HAQ walking score 1.63 , 95\% CI $1.53-$ 


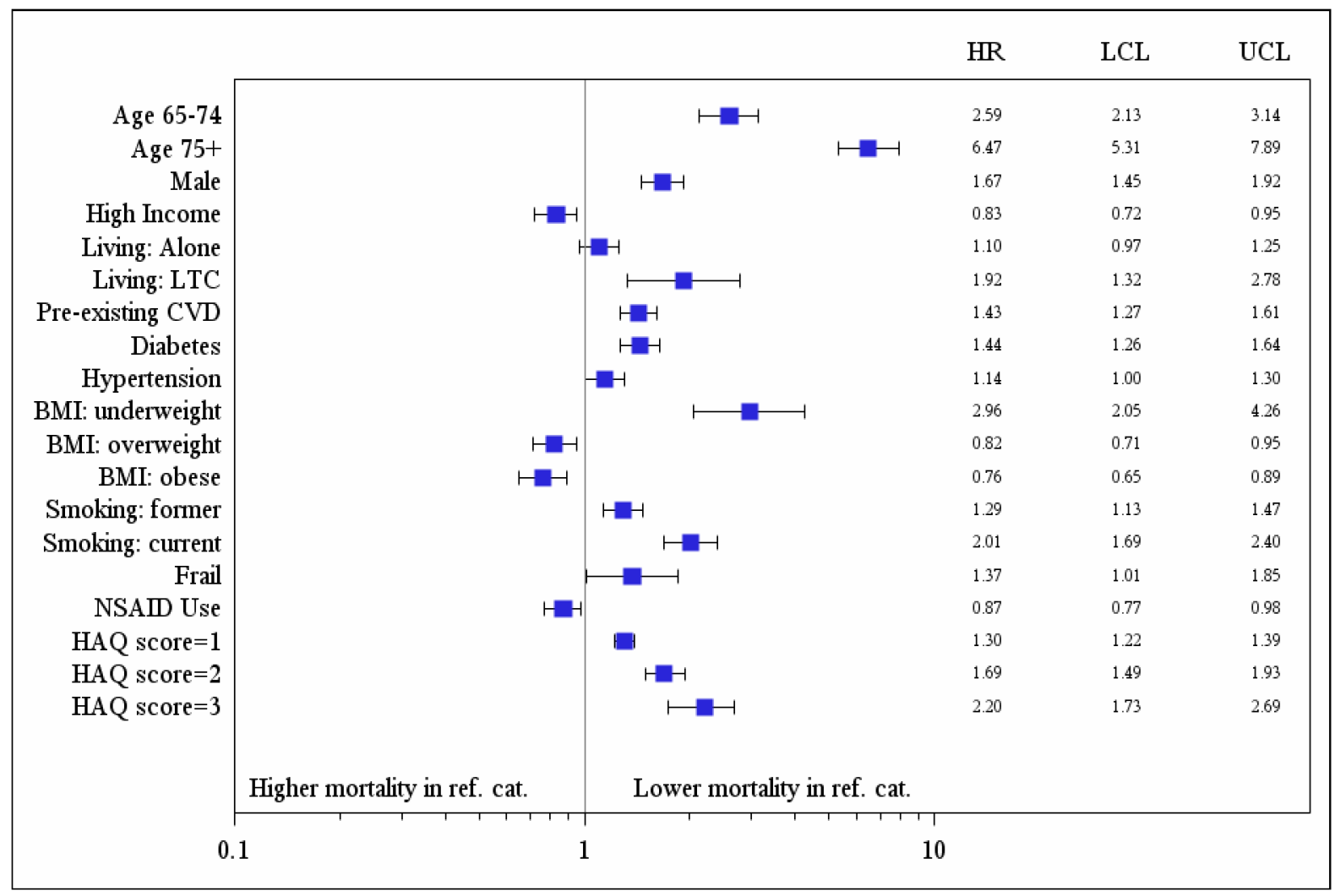

Figure 2. Results of multivariable analysis: independent predictors of all-cause mortality. doi:10.1371/journal.pone.0091286.g002

$1.74, \mathrm{p}<0.001$; HR for use of walking aids $2.28,95 \%$ CI $2.04-$ $2.55, \mathrm{p}<0.001$; HR per 10-point increase in WOMAC pain score $1.00,95 \%$ CI $0.98-1.03, \mathrm{p}=0.85)$. Additional predictors of all cause mortality are shown in Table 2. Other predictors of higher mortality in the multivariable model were older age, male sex, lower income, nursing home residence, pre-existing CVD, diabetes, hypertension, being underweight, smoking and frailty; self-reported NSAID use and being overweight or obese were protective. Controlling for these factors, all-cause mortality was independently predicted by higher (worse) baseline WOMAC function scores (adjusted HR per 10-point increase in score 1.04, $95 \%$ CI $1.01-1.07, \mathrm{p}=0.004$ ) and by walking disability (adjusted HR per unit increase in the HAQ walking score $1.30,95 \%$ CI $1.22-1.39, \mathrm{p}<0.001$; use of a walking aid 1.51, 95\% CI 1.34-1.70, $\mathrm{p}<0.0001)$. Model fit was best with the HAQ walking score (explained variance 0.57) [30] (Figures 2 and 3). There were no significant interactions between pre-existing CVD and baseline OA disability (HAQ walking score, $\mathrm{p}=0.31$; use of an aid, $\mathrm{p}=0.64$; WOMAC function, $\mathrm{p}=0.70$ ). However, there was a significant interaction between pre-existing CVD and baseline WOMAC pain score $(p=0.009)$; greater baseline OA pain predicted higher mortality in those with, but not without, preexisting CVD (adjusted HR per 10-point increase in WOMAC pain score $=1.04,95 \%$ CI $1.00-1.08, \mathrm{p}=0.038$ and $0.97,95 \%$ CI $0.93-1.01, \mathrm{p}=0.96$, respectively).

\section{Effect of Baseline OA Pain and Disability on Time to \\ Composite CVD Outcome}

Over a median 9.2 years of follow-up (IQR 3.7-15.0 years), 822 (38.1\%) experienced one or more CVD events (193 AMI, 292
CHF, 288 TIA/stroke, and 71 CABG/PCI). Censoring on death, time to occurrence of the first CVD event was reduced in those with greater baseline OA disability (crude HR per 10-point increase in WOMAC function score $=1.10,95 \%$ CI $1.05-1.16$, $\mathrm{p}<0.001$; crude HR per unit increase in HAQ walking score $=1.40 ; 95 \%$ CI $1.30-1.51, \mathrm{p}<0.001$; crude HR for use of walking aids $1.75,95 \%$ CI $1.53-2.01, \mathrm{p}<0.001$ ), but unrelated to baseline OA pain (crude HR per 10-point increase in WOMAC pain score $=1.08 ; 95 \%$ CI $0.92-1.07, \mathrm{p}=0.34)$. Additional significant predictors are shown in Table 3. In our final model, risk of a CVD outcome was significantly associated with older age, male sex, pre-existing CVD, diabetes, hypertension, smoking, and with walking disability (adjusted HR per unit increase in HAQ walking score $=1.17,95 \%$ CI 1.08-1.27, $\mathrm{p}<0.001$; adjusted HR for baseline use of a walking aid $=1.28,95 \%$ CI $1.11-1.48, \mathrm{p}<0.001$ ) (Table 4). We found no significant interactions between baseline OA symptom severity measures and pre-existing CVD (data not shown).

\section{Secondary Analyses}

When we restricted our analyses to the 1,501 participants who were $\geq 66$ years at baseline, and thus had been eligible for drug benefits for at least one year, and further adjusted for receipt of a prescription for a non-ASA NSAID, our results were unchanged (data not shown).

Among the 2,156 OA cohort participants, 402 (18.65\%) received a post-baseline primary, elective hip or knee TJA prior to death or censoring. Of these, most (93.8\%) received their TJA prior to experiencing a GVD event or censoring. Additional adjustment of our final models for receipt of a post-baseline primary, elective TJA found that TJA was protective of both our 
Table 2. Sample Characteristics at Baseline by Occurrence of All-Cause Death (values are numbers and percentages unless otherwise stated).

\begin{tabular}{|c|c|c|c|c|}
\hline \multirow[t]{2}{*}{ Predictor Variables } & \multicolumn{2}{|c|}{ Participant Died } & \multirow[t]{2}{*}{$\begin{array}{l}\text { Crude Hazard Ratio*(95\% } \\
\text { confidence interval) }\end{array}$} & \multirow[t]{2}{*}{ p-value* } \\
\hline & No $(N=920)$ & Yes $(N=1236)$ & & \\
\hline \multicolumn{3}{|l|}{ Age: } & & $<0.001$ \\
\hline 55-64 (reference) & $445(48.4 \%)$ & $146(11.8 \%)$ & 1.0 & \\
\hline $65-74$ & $365(39.7 \%)$ & 402 (32.5\%) & $2.60(2.15-3.14)$ & \\
\hline $75+$ & $110(12.0 \%)$ & $688(55.7 \%)$ & $7.11(5.94-8.52)$ & \\
\hline Male sex & $215(23.4 \%)$ & 389 (31.5\%) & $1.34(1.19-1.52)$ & $<0.001$ \\
\hline \multicolumn{3}{|l|}{ Income: } & & $<0.001$ \\
\hline Less than $\$ 20 \mathrm{~K}$ (reference) & $440(47.8 \%)$ & $692(56.0 \%)$ & 1.0 & \\
\hline Greater than $\$ 20 \mathrm{~K}$ & $324(35.2 \%)$ & $325(26.3 \%)$ & $0.73(0.64-0.84)$ & $<0.001$ \\
\hline Missing & $156(17.0 \%)$ & $219(17.7 \%)$ & $0.92(0.79-1.07)$ & 0.28 \\
\hline \multicolumn{3}{|l|}{ Education: } & & $<0.001$ \\
\hline Less than high school (reference) & $294(32.0 \%)$ & $484(39.2 \%)$ & 1.0 & \\
\hline High school graduation & $451(49.0 \%)$ & $562(45.5 \%)$ & $0.81(0.72-0.92)$ & $<0.001$ \\
\hline Post-secondary education & $165(17.9 \%)$ & $162(13.1 \%)$ & $0.70(0.58-0.83)$ & $<0.001$ \\
\hline Missing & $10(1.1 \%)$ & $28(2.3 \%)$ & $1.29(0.88-1.89)$ & 0.19 \\
\hline \multicolumn{2}{|l|}{ Living arrangements: } & & & $<0.001$ \\
\hline Lives with others (reference) & $691(75.1 \%)$ & $734(59.4 \%)$ & 1.0 & \\
\hline Lives alone & $220(23.9 \%)$ & $451(36.5 \%)$ & $1.54(1.37-1.73)$ & $<0.001$ \\
\hline Living in long term care residence & $2(0.2 \%)$ & $31(2.5 \%)$ & $4.42(3.08-6.34)$ & $<0.001$ \\
\hline Missing & $7(0.8 \%)$ & $20(1.6 \%)$ & $1.89(1.21-2.95)$ & 0.005 \\
\hline Urban place of residence & $386(42.0 \%)$ & $556(45.0 \%)$ & $1.07(0.96-1.20)$ & 0.21 \\
\hline Pre-existing CVD risk & $228(24.8 \%)$ & $626(50.7 \%)$ & $2.11(1.89-2.36)$ & $<0.001$ \\
\hline Diabetes & $126(13.7 \%)$ & $314(25.4 \%)$ & $1.64(1.44-1.86)$ & $<0.001$ \\
\hline Hypertension & $506(55.0 \%)$ & $863(69.8 \%)$ & $1.54(1.37-1.74)$ & $<0.001$ \\
\hline $\begin{array}{l}\text { Depression } \\
\text { (SF36-mental health subscale) }\end{array}$ & $243(26.4 \%)$ & $378(30.6 \%)$ & $1.18(1.05-1.33)$ & 0.007 \\
\hline \multicolumn{2}{|l|}{ No. other comorbid conditions: } & & $1.11(1.04-1.18)$ & 0.003 \\
\hline 0 & $37(4.0 \%)$ & $47(3.8 \%)$ & & \\
\hline 1 & $176(19.1 \%)$ & $214(17.3 \%)$ & & \\
\hline 2 & $314(34.1 \%)$ & $364(29.5 \%)$ & & \\
\hline $3+$ & 393 (42.7\%) & $611(49.4 \%)$ & & \\
\hline Frailty & $6(0.7 \%)$ & $48(3.9 \%)$ & $2.67(2.00-3.56)$ & $<0.001$ \\
\hline \multicolumn{2}{|l|}{ Body mass index (BMI): } & & & $<0.001$ \\
\hline Underweight & $3(0.3 \%)$ & $33(2.7 \%)$ & $3.23(2.26-4.63)$ & $<0.001$ \\
\hline Normal (reference) & $208(22.6 \%)$ & $338(27.4 \%)$ & 1.0 & \\
\hline Overweight & $322(365.0 \%)$ & $439(35.5 \%)$ & $0.84(0.72-0.96)$ & 0.013 \\
\hline Obese & $361(39.2 \%)$ & $322(26.1 \%)$ & $0.54(0.54-0.73)$ & $<0.001$ \\
\hline Missing & $26(2.8 \%)$ & $104(8.4 \%)$ & $1.73(1.39-2.16)$ & $<0.001$ \\
\hline \multicolumn{2}{|l|}{ Smoking history: } & & & 0.049 \\
\hline Never smoked (reference) & $453(49.2 \%)$ & $559(45.2 \%)$ & 1.0 & \\
\hline Former smoker & $331(36.0 \%)$ & $452(36.6 \%)$ & $1.08(0.95-1.22)$ & 0.23 \\
\hline Current smoker & $119(12.9 \%)$ & $193(15.6 \%)$ & $1.24(1.05-1.46)$ & 0.010 \\
\hline Missing & $17(1.9 \%)$ & $32(2.6 \%)$ & $1.29(0.90-1.84)$ & 0.16 \\
\hline $\begin{array}{l}\text { No. visits to a primary care } \\
\text { physician in pre-baseline year: } \\
\text { median }(\mathrm{IQR})^{\dagger}\end{array}$ & $6(3-10)$ & $7(4-12)$ & $1.13(1.08-1.19)$ & $<0.001$ \\
\hline $\begin{array}{l}\text { No. visits to a specialist in } \\
\text { pre-baseline year: median (IQR) }\end{array}$ & $2(0-5)$ & $3(1-7)$ & $1.12(1.08-1.17)$ & $<0.001$ \\
\hline WOMAC Scores: Mean (SD) $)^{\ddagger}$ & & & & \\
\hline
\end{tabular}


Table 2. Cont.

\begin{tabular}{|c|c|c|c|c|}
\hline \multirow[t]{2}{*}{ Predictor Variables } & \multicolumn{2}{|c|}{ Participant Died } & \multirow[t]{2}{*}{$\begin{array}{l}\text { Crude Hazard Ratio*(95\% } \\
\text { confidence interval) }\end{array}$} & \multirow[t]{2}{*}{ p-value* } \\
\hline & No $(N=920)$ & Yes $(N=1236)$ & & \\
\hline Physical function/100 & $38.1(20.0)$ & $42.4(21.2)$ & $1.08(1.05-1.11)$ & $<0.001$ \\
\hline Pain/100 & $41.0(20.9)$ & $40.8(22.5)$ & $1.00(0.98-1.03)$ & 0.85 \\
\hline HAQ walking score (/3): mean (IQR) & $1(0-2)$ & $2(1-2)$ & $1.63(1.53-1.74)$ & $<0.001$ \\
\hline Uses an aid to walk & $953(44.2 \%)$ & $692(56.0 \%)$ & $2.28(2.04-2.55)$ & $<0.001$ \\
\hline Self-reported NSAID use & $357(38.8 \%)$ & 459 (37.1\%) & $0.90(0.80-1.01)$ & 0.080 \\
\hline
\end{tabular}

primary and secondary outcomes (adjusted HR for all-cause death $=0.62,95 \%$ CI $0.51-0.76, \mathrm{p}<0.001$; adjusted HR for CVD events $=0.66,95 \%$ CI $0.52-0.85, \mathrm{p}<0.001$ ), but did not affect the adjusted HRs for baseline measures of OA pain or disability (data not shown).

\section{Discussion}

This population-based cohort study of persons with hip and knee OA followed for over a decade found a significant association between greater OA-related disability and both all-cause mortality and risk for a serious CVD event. Survival was also reduced among those with pre-existing CVD who had greater baseline OA pain. Our findings are important. As the proportion of the population that is affected by multiple chronic conditions increases, it is critical to understand the impact of comorbidity on health outcomes. Among the most common disease dyads is osteoarthritis and ischemic heart disease, estimated to affect one in five Medicare beneficiaries [12]. Our findings therefore have substantial implications for clinical practice and quality improvement to optimize outcomes in people with $\mathrm{OA}$ and heart disease.

Our results confirm those of Nuesch et al [19], who documented increased all-cause mortality in individuals with hip and knee OA relative to the age- and sex-matched population of England, and linked increased mortality risk with walking disability. Additionally, our results extend their work in showing that within individuals

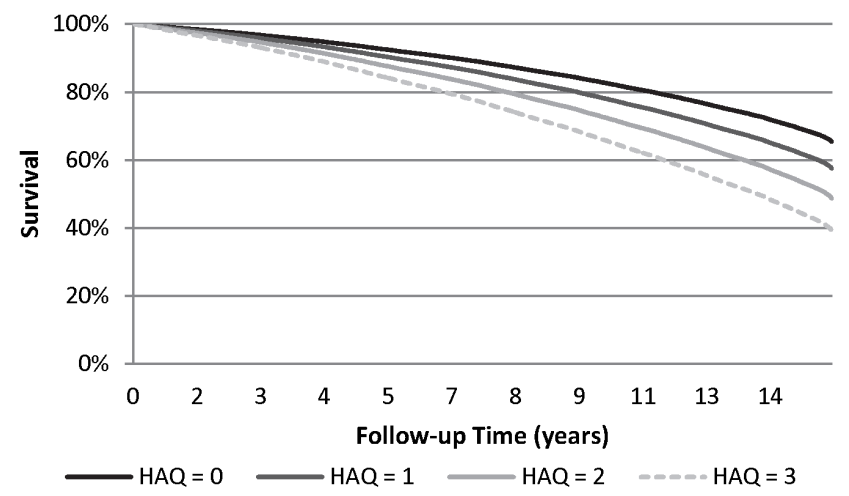

Figure 3. Survival curves for the typical osteoarthritis (OA) cohort participant* by level of walking disability (HAQ walking score) at baseline assessment. *typical defined as the mean or most common value for variables in the final model for all-cause death. doi:10.1371/journal.pone.0091286.g003 with hip and knee $O A$, severity of OA-related pain and disability are significant predictors of future risk for all-cause death. These effects remained after controlling for multiple potential confounders, including obesity and obesity-related conditions, social health determinants and mental health status. Of the OA severity measures assessed, walking disability assessed at baseline using the two-item HAQ walking scale had the greatest ability to predict survival such that survival at the end of our follow-up period was approximately 25\% lower among those with the highest (worse) versus lowest scores for walking disability at baseline. Further research is warranted to elucidate the mechanisms by which reduced mobility due to $\mathrm{OA}$ impacts survival.

The prior study by Nuesch et al found that increased mortality in people with OA was largely due to CVD causes. As noted above, OA-related disability may increase cardiac risk by limiting ability to engage in physical activity and decreasing cardiorespiratory fitness $[16,31,32]$. Thus, we tested for an independent effect of baseline OA disability on risk for serious CVD events. Controlling for multiple potential confounders, we found that risk for our composite CVD outcome was almost 30\% higher among those with versus without self-reported use of walking aids. Further research is warranted to elucidate the extent to which increased risk for GVD events due to walking disability contributes to reduced survival in people with hip and knee OA. We also observed a positive significant relationship between baseline OA pain severity and all-cause death among those with pre-existing CVD; this finding is intriguing and warrants further study.

Two-thirds of our OA participants were overweight or obese, two-thirds had hypertension, almost forty percent had pre-existing GVD, and one-fifth had diabetes. In such individuals, functional limitations due to OA may impact ability to participate in physical activity, contributing to weight gain and physical deconditioning, worse disease control, and increased risk for adverse outcomes including death. Although, to date, no study has explicitly evaluated the contribution of $\mathrm{OA}$ to the management and outcomes of other common chronic conditions, there is indirect support for a relationship. In a survey of three groups of mainly male US Veterans aged 66-74 years (heart failure; diabetes; and general primary care users), activity limitations due to chronic pain were commonly reported ( $60 \%$ in all groups). Most reported back, hip or knee pain [33]. Of the $60 \%$ who reported pain limiting activities, most were managing their pain with rest or sedentary activities (e.g. watching TV) and fewer than half were exercising. Collectively, these findings underscore the need to move away from single-disease focused clinical practice guidelines towards 
Table 3. Sample characteristics at baseline by occurrence of a subsequent composite cardiovascular outcome.

\begin{tabular}{|c|c|c|c|c|}
\hline \multirow[t]{2}{*}{ Predictor Variables } & \multicolumn{2}{|c|}{$\begin{array}{l}\text { Participant Experienced Composite } \\
\text { CVD Outcome }\end{array}$} & \multirow[t]{2}{*}{$\begin{array}{l}\text { Crude Hazard Ratio* } \\
\text { ( } 95 \% \text { confidence interval) }\end{array}$} & \multirow[t]{2}{*}{ p-value } \\
\hline & No $(N=1,334)$ & Yes $(\mathbf{N}=\mathbf{8 2 2})$ & & \\
\hline Age category: N (\%) & & & & $<0.001$ \\
\hline 55-64 (reference) & 447 (33.5\%) & $144(17.5 \%)$ & 1.0 & \\
\hline $65-74$ & 457 (34.3\%) & $310(37.7 \%)$ & $2.07(1.70-2.52)$ & $<0.001$ \\
\hline $75+$ & $430(32.2 \%)$ & $368(44.8 \%)$ & $3.55(2.92-4.32)$ & $<0.001$ \\
\hline Male sex: $\mathrm{N}(\%)$ & $360(27.0 \%)$ & $244(29.7 \%)$ & $1.27(1.09-1.48)$ & 0.002 \\
\hline Income : N (\%) & & & & 0.0036 \\
\hline Less than $\$ 20 \mathrm{~K}$ (reference) & $673(50.5 \%)$ & $459(55.8 \%)$ & 1.0 & \\
\hline Greater than $\$ 20 \mathrm{~K}$ & $430(32.2 \% 0$ & $219(26.6 \%)$ & $0.76(0.65-0.89)$ & 0.0008 \\
\hline Missing & $231(17.3 \%)$ & $144(17.5 \%)$ & $0.90(0.74-1.08)$ & 0.90 \\
\hline Education: N (\%) & & & & 0.001 \\
\hline Less than high school (reference) & $458(34.3 \%)$ & $320(38.9 \%)$ & 1.0 & \\
\hline High school graduation & $641(48.1 \%)$ & $372(45.3 \%)$ & $0.82(0.71-0.95)$ & 0.009 \\
\hline Post-secondary education & $217(16.3 \%)$ & $110(13.4 \%)$ & $0.73(0.59-0.90)$ & 0.004 \\
\hline Missing & $18(1.4 \%)$ & $20(2.4 \%)$ & $1.41(0.90-2.21)$ & 0.14 \\
\hline Living arrangements: N (\%) & & & & $<0.001$ \\
\hline Lives with others (reference) & 897 (67.2\%) & $528(64.2 \%)$ & 1.0 & \\
\hline Lives alone & $401(30.1 \%)$ & $270(32.9 \%)$ & $1.24(1.07-1.43)$ & 0.005 \\
\hline Long term care residence & $19(1.4 \%)$ & $14(1.7 \%)$ & $2.53(1.48-4.30)$ & $<0.001$ \\
\hline Missing & $17(1.3 \%)$ & $10(1.2 \%)$ & $1.18(0.63-2.21)$ & 0.60 \\
\hline Urban place of residence: $\mathrm{N}(\%)$ & $585(43.9 \%)$ & $357(43.4 \%)$ & $1.01(0.88-1.16)$ & 0.91 \\
\hline Pre-existing CVD: N (\%) & $425(31.9 \%)$ & $429(52.2 \%)$ & $2.42(2.11-2.77)$ & $<0.001$ \\
\hline Diabetes: N (\%) & 209 (15.7\%) & $231(28.1 \%)$ & $2.01(1.72-2.34)$ & $<0.001$ \\
\hline Hypertension: N (\%) & $786(58.9 \%)$ & $583(70.9 \%)$ & $1.68(1.44-1.95)$ & $<0.001$ \\
\hline $\begin{array}{l}\text { Depression } \\
\text { (SF36-mental health subscale) : N (\%) }\end{array}$ & $380(28.5 \%)$ & $241(29.3 \%)$ & $1.11(0.95-1.28)$ & 0.19 \\
\hline No. other comorbid conditions ${ }^{\dagger}$ & & & $1.07(1.01-1.14)$ & 0.025 \\
\hline 0 & $46(3.5 \%)$ & $38(4.6 \%)$ & & \\
\hline 1 & $257(19.3 \%)$ & $133(16.2 \%)$ & & \\
\hline 2 & $426(31.9 \%)$ & $252(30.7 \%)$ & & \\
\hline $3+$ & $605(45.4 \%)$ & $399(48.5 \%)$ & & \\
\hline Frailty: N (\%) & $30(2.3 \%)$ & $24(2.9 \%)$ & $1.85(1.23-2.77)$ & 0.003 \\
\hline Body mass index (BMI): N (\%) & & & & $<0.001$ \\
\hline Underweight & $26(2.0 \%)$ & $10(1.2 \%)$ & $1.37(0.89-1.28)$ & 0.33 \\
\hline Normal (reference) & $359(26.9 \%)$ & $187(22.8 \%)$ & 1.0 & \\
\hline Overweight & 465 (34.9\%) & $296(36.0 \%)$ & $1.06(0.89-1.28)$ & 0.50 \\
\hline Obese & 415 (31.1\%) & $268(32.6 \%)$ & $1.02(0.85-1.23)$ & 0.85 \\
\hline Missing & $69(5.2 \%)$ & $61(7.4 \%)$ & $1.85(1.39-2.48)$ & $<0.001$ \\
\hline Smoking history: N (\%) & & & & 0.19 \\
\hline Never smoked (reference) & $629(47.2 \%)$ & $383(46.6 \%)$ & 1.0 & \\
\hline Former smoker & $481(36.1 \%)$ & 302 (36.7\%) & $1.07(0.92-1.24)$ & 0.42 \\
\hline Current smoker & $200(15.0 \%)$ & 112 (5.2\%) & $1.01(0.82-1.24)$ & 0.95 \\
\hline Missing & $24(1.8 \%)$ & $25(3.0 \%)$ & $1.55(1.03-2.32)$ & 0.035 \\
\hline $\begin{array}{l}\text { No. visits to a primary care physician in } \\
\text { pre-baseline year: median }(\mathrm{IQR})^{\ddagger}\end{array}$ & $6(3-11)$ & $7(4-11)$ & $1.18(1.11-1.25)$ & $<0.001$ \\
\hline $\begin{array}{l}\text { No. visits to a specialist in } \\
\text { pre-baseline year: median (IQR) }\end{array}$ & $2(0-6)$ & $3(1-6)$ & $1.10(1.05-1.15)$ & $<0.001$ \\
\hline \multicolumn{5}{|l|}{ WOMAC Scores: Mean (SD) ${ }^{\S}$} \\
\hline Physical function/100 & $39.8(20.5)$ & $41.7(21.2)$ & $1.10(1.05-1.16)$ & $<0.001$ \\
\hline
\end{tabular}


Table 3. Cont.

\begin{tabular}{|c|c|c|c|c|}
\hline \multirow[t]{2}{*}{ Predictor Variables } & \multicolumn{2}{|c|}{$\begin{array}{l}\text { Participant Experienced Composite } \\
\text { CVD Outcome }\end{array}$} & \multirow[t]{2}{*}{$\begin{array}{l}\text { Crude Hazard Ratio* } \\
\text { (95\% confidence interval) }\end{array}$} & \multirow[t]{2}{*}{ p-value } \\
\hline & No $(N=1,334)$ & Yes $(\mathrm{N}=\mathbf{8 2 2})$ & & \\
\hline Pain/100 & $40.7(21.5)$ & $41.2(22.5)$ & $1.08(0.92-1.27)$ & 0.34 \\
\hline HAQ walking score/3: median (IQR) & $1(0-2)$ & $2(1-2)$ & $1.40(1.30-1.51)$ & $<0.001$ \\
\hline Use of aid for walking: $\mathrm{N}(\%)$ & $542(40.6 \%)$ & 411 (50.0\%) & $1.75(1.53-2.01)$ & $<0.001$ \\
\hline Self-reported NSAID use: N (\%) & $494(37.0 \%)$ & $322(39.2 \%)$ & $1.03(0.90-1.19)$ & 0.65 \\
\hline Number of troublesome hips/knees: $\mathrm{N}(\%)$ & & & $0.95(0.90-1.00)$ & 0.050 \\
\hline 0 & $212(15.9 \%)$ & $145(17.6 \%)$ & & \\
\hline 1 & $197(14.8 \%)$ & $113(13.8 \%)$ & & \\
\hline 2 & $493(37.0 \%)$ & $293(35.6 \%)$ & & \\
\hline 3 & 139 910.4\%) & $110(13.4 \%)$ & & \\
\hline 4 & $293(22.0 \%)$ & $161(19.6 \%)$ & & \\
\hline
\end{tabular}

*Univariable hazard ratios, $95 \%$ confidence intervals and $\mathrm{P}$ values were derived from a survival model, censoring on death; missing baseline data were either imputed from responses on the year 1 follow-up assessment or incorporated as a 'missing' dummy variable. Hazard ratios $>1$ indicate lower mortality in reference category. Hazard ratio is per additional comorbid condition.

${ }^{\ddagger}$ Hazard ratios are per doubling of the number of visits in the pre-baseline year.

${ }^{\S}$ Hazard ratios are per 10-point increase in WOMAC subscale score.

doi:10.1371/journal.pone.0091286.t003

chronic disease strategies that consider the potential for common approaches to chronic disease prevention and management, including targeted interventions to address mobility.

Total joint arthroplasty (TJA) of the hip and knee is a highly effective treatment for advanced hip and knee OA[34-36]. Thus, if walking disability increases risk for CVD events or death, receipt of a TJA procedure may be protective. Indeed, in a secondary analysis, we found that post-baseline receipt of a primary, elective hip or knee TJA was associated with improved survival and reduced risk for CVD events, but did not affect the observed relationships between measures of OA disability and our outcomes. Controlling for other factors, those who received

Table 4. Results of multivariable survival analysis: effect of baseline osteoarthritis (OA) pain and disability on risk for composite cardiovascular (CVD) outcome after adjusting for covariates and censoring on death.

\begin{tabular}{|c|c|c|c|}
\hline Participant Characteristic & Adjusted Hazard Ratio & 95\% confidence interval & p-value \\
\hline Age: & & & $<0.001$ \\
\hline 55-64 (reference) & 1.0 & & \\
\hline $65-74$ & 1.90 & $1.55-2.33$ & $<0.001$ \\
\hline $75+$ & 3.05 & $2.46-3.78$ & $<0.001$ \\
\hline Male (female is reference) & 1.31 & $1.11-1.54$ & 0.001 \\
\hline Pre-existing cardiovascular disease & 1.86 & $1.61-2.15$ & $<0.001$ \\
\hline Diabetes & 1.67 & $1.43-1.96$ & $<0.001$ \\
\hline Hypertension & 1.20 & $1.02-1.40$ & 0.026 \\
\hline Body mass index (BMI): & & & 0.20 \\
\hline Underweight & 1.36 & $0.72-2.58$ & 0.35 \\
\hline Normal (reference) & 1.0 & & \\
\hline Overweight & 1.07 & $0.89-1.29$ & 0.49 \\
\hline Obese & 1.11 & $0.92-1.35$ & 0.28 \\
\hline Missing & 1.42 & $1.06-1.92$ & 0.021 \\
\hline Smoking history: & & & 0.016 \\
\hline Never smoked (reference) & 1.0 & & \\
\hline Former smoker & 1.16 & $0.98-1.36$ & 0.080 \\
\hline Current smoker & 1.38 & $1.10-1.72$ & 0.004 \\
\hline Missing & 1.47 & $0.95-2.27$ & 0.083 \\
\hline HAQ walking score (per unit increase in score) & 1.17 & $1.08-1.27$ & $<0.001$ \\
\hline
\end{tabular}

Model R-square $=0.381$.

doi:10.1371/journal.pone.0091286.t004 
a post-baseline primary, elective TJA were approximately one third less likely to experience our outcomes. While these findings are intriguing, we cannot rule out a 'healthy user effect' as the explanation for these results. In subsequent research using our cohort data, we therefore examined the relationship between receipt of hip or TJA and risk for CVD events using a propensity score matched landmark analysis [37]. Using this approach, TJA remained significantly and independently protective of risk for CVD events. Further research is warranted to elucidate explanations for this relationship, including the role of physical activity, pain relief, and reduction in use of NSAIDs.

Strengths of our study include reliance on an established, well-characterized population-based cohort with hip and knee OA, long duration of follow-up, ascertainment of survival status of all subjects regardless of post-baseline survey participation, and validated outcomes using linked health administrative data. However, there are also some study limitations. First, we were unable to control for lipid levels, abdominal obesity and family history of GVD and we did not consider the effect of medication use to manage CVD risk, such as anti-hypertensive and lipid lowering drugs. Thus, lack of control for unmeasured confounders remains a possible explanation for our findings. Second, we did not control for changes in OA symptom severity - or other covariates, such as CVD status - over the follow-up period as we were interested in the prognostic value of measures of OA disability on our outcomes of interest and participation

\section{References}

1. World Health Organization (2012) World Health Statistics 2012. Geneva: World Health Organization.

2. Statistics Canada (2011) Health Trends. Statistics Canada Catalogue No. 82 213-XWE. Ottawa. Available: http://www12.statcan.gc.ca/health-sante/82213/index.cfm?Lang = Eng. Accessed 2013 July 15.

3. Tu K, Chen Z, Lipscombe LL (2008) Prevalence and incidence of hypertension from 1995 to 2005: a population-based study. CMAJ 178: 1429-1435.

4. Tu JV, Nardi L, Fang J, Liu J, Khalid L, et al. (2009) National trends in rates of death and hospital admissions related to acute myocardial infarction, heart failure and stroke, 1994-2004. CMAJ 180: E118-E125.

5. Vinluan CM, Zreikat HH, Levy JR, Cheang KI (2012) Comparison of different metabolic syndrome definitions and risks of incident cardiovascular events in the elderly. Metabolism 61: 302-309.

6. Lipscombe LL, Hux JE (2007) Trends in diabetes prevalence, incidence, and mortality in Ontario, Canada 1995-2005: a population-based study. Lancet 369: $750-756$.

7. Lee DS, Chiu M, Manuel DG, Tu K, Wang X, et al. (2009) Trends in risk factors for cardiovascular disease in Canada: temporal, socio-demographic and geographic factors. CMAJ 181: E55-E66.

8. Perruccio AV, Power JD, Badley EM (2006) Revisiting arthritis prevalence projections-it's more than just the aging of the population. J Rheumatol 33: 1856-1862.

9. Freedman VA, Martin LG (2000) Contribution of chronic conditions to aggregate changes in old-age functioning. Am J Public Health 90: 1755-1760.

10. Hawker GA, Gignac MA, Badley E, Davis AM, French MR, et al. (2011) A longitudinal study to explain the pain-depression link in older adults with osteoarthritis. Arthritis Care Res (Hoboken) 63: 1382-1390.

11. Zhang W, Nuki G, Moskowitz RW, Abramson S, Altman RD, et al. (2010) OARSI recommendations for the management of hip and knee osteoarthritis: part III: Changes in evidence following systematic cumulative update of research published through January 2009. Osteoarthritis Cartilage 18: 476-499.

12. Centers for Medicare and Medicaid Services (2012) Chronic Conditions among Medicare Beneficiaries, Chartbook, 2012 Edition. Baltimore, MD: CMS.

13. Trelle S, Reichenbach S, Wandel S, Hildebrand P, Tschannen B, et al. (2011) Cardiovascular safety of non-steroidal anti-inflammatory drugs: network metaanalysis. BMJ 342: c7086.

14. Hackam DG, Khan NA, Hemmelgarn BR, Rabkin SW, Touyz RM, et al. (2010) The 2010 Canadian Hypertension Education Program recommendations for the management of hypertension: part 2 - therapy. Can J Cardiol 26: 249 258.

15. Sale JEM, Gignac M, Hawker G (2006) How "bad" does the pain have to be? A qualitative study examining adherence to pain medication in older adults with osteoarthritis. Arthritis Rheum 55: 272-278. attrition over time would have reduced our sample size and thus power. This approach may have resulted in misclassification bias. We have previously shown that, in the absence of TJA, OA disability, as measured using the WOMAC, worsens over time [28]. Thus, we may have underestimated the estimated effect of OA symptom severity on our outcomes of interest.

In conclusion, controlling for other factors, our study identified hip/knee OA functional limitations - and specifically walking disability - as a potentially modifiable risk factor for serious CVD events and death. Pragmatic clinical trials of interventions to reduce walking disability in individuals with multi-morbidity, including OA, are needed. Chronic disease strategies that target combinations of clustered conditions - and recognize the impact of OA - are likely to be more effective and are needed.

\section{Acknowledgments}

These data sets were held securely in a linked, de-identified form and analyzed at the Institute for Clinical Evaluative Sciences.

\section{Author Contributions}

Conceived and designed the experiments: GH RC AB PH BR IS LL. Performed the experiments: RC GH. Analyzed the data: RC. Wrote the paper: GH IS. Critical review of the manuscript: GH RC AB PH BR IS LL. Final approval of manuscript to be published: GH RC AB PH BR IS LL

16. Kodama S, Saito K, Tanaka S, Maki M, Yachi Y, et al. (2009) Cardiorespiratory fitness as a quantitative predictor of all-cause mortality and cardiovascular events in healthy men and women: a meta-analysis. JAMA 301: 2024-2035.

17. Gulati M, Black HR, Shaw LJ, Arnsdorf MF, Merz CN, et al. (2005) The prognostic value of a nomogram for exercise capacity in women. N Engl J Med 353: $468-475$.

18. Bodenheimer T, Lorig K, Holman H, Grumbach K (2002) Patient selfmanagement of chronic disease in primary care. JAMA 288: 2469-2475.

19. Nuesch E, Dieppe P, Reichenbach S, Williams S, Iff S, et al. (2011) All cause and disease specific mortality in patients with knee or hip osteoarthritis: population based cohort study. BMJ 342: d1165. doi: 10.1136/bmj.d1 165 .

20. Hawker GA, Wright JG, Coyte PC, Williams JI, Harvey B, et al. (2001) Determining the need for hip and knee arthroplasty: the role of clinical severity and patients' preferences. Med Care 39: 206-16.

21. Friedman B, Heisel M, Delavan R (2005) Validity of the SF-36 five-item mental health index for major depression in functionally impaired, community-dwelling elderly patients. J Am Geriatr Soc 53: 1978-85.

22. Bellamy N, Buchanan WW, Goldsmith CH, Campbell J, Stitt LW (1988) Validation study of WOMAC: a health status instrument for measuring clinically important patient relevant outcomes to antirheumatic drug therapy in patients with osteoarthritis of the hip or knee. J Rheumatol 15: 1833-40.

23. Fries JF, Spitz P, Kraines RG, Holman HR (1980) Measurement of patient outcome in arthritis. Arthritis Rheum 23: 137-145.

24. Tu K, Campbell NR, Chen ZL, Cauch-Dudek KJ, McAlister FA (2007) Accuracy of administrative databases in identifying patients with hypertension. Open Med 1: e18-e26.

25. Hux JE, Ivis F, Flintoft V, Bica A (2002) Diabetes in Ontario: determination of prevalence and incidence using a validated administrative data algorithm. Diabetes Care 25: 512-516.

26. Alter DA, Chong A, Austin PC, Mustard C, Iron K, et al. (2006) Socioeconomic status and mortality after acute myocardial infarction. Ann Intern Med 144: 82 93.

27. Weiner JP (2011) The Johns Hopkins ACG $^{\circledR}$ System: Technical Reference Guide, Version 10.0. Baltimore, MD: Johns Hopkins University.

28. Hawker GA, Badley EM, Croxford R, Coyte PC, Glazier RH, et al. (2009) A Population Based Nested Case-Control Study of the Costs of Hip and Knee Replacement Surgery. Medical Care 47: 732-741.

29. Leffondre K, Abrahamowicz M, Regeasse A, Hawker GA, Badley EM, et al. (2004) Statistical measures were proposed for identifying longitudinal patterns of change in quantitative health indicators. J Clin Epidemiol 57: 1049-1062.

30. Choodari-Oskooei B, Royston P, Parmar MK (2012) A simulation study of predictive ability measures in a survival model I: explained variation measures. Stat Med 31: 2627-2643

31. Haskell WL, Lee IM, Pate RR, Powell KE, Blair SN, et al. (2007) Physical activity and public health: updated recommendation for adults from the 
American College of Sports Medicine and the American Heart Association. Circulation 116: 1081-1093.

32. Thompson PD, Buchner D, Pina IL, Balady GJ, Williams MA, et al. (2003) Exercise and physical activity in the prevention and treatment of atherosclerotic cardiovascular disease: a statement from the Council on Clinical Cardiology (Subcommittee on Exercise, Rehabilitation, and Prevention) and the Council on Nutrition, Physical Activity, and Metabolism (Subcommittee on Physical Activity). Circulation 107: 3109-3116.

33. Butchart A, Kerr EA, Heisler M, Piette JD, Krein SL (2009) Experience and management of chronic pain among patients with other complex chronic conditions. Clin J Pain 25: 293-298.
34. Gunther KP (2001) Surgical approaches for osteoarthritis. Best Pract Res Clin Rheumatol 15: 627-643.

35. Paterson MJ, DeBoer DP, Williams J, Bourne RB, Hawker G, et al. (2006) Total Hip and Knee Replacement. Access to Health Services in Ontario. ICES Atlas.

36. NIH Consensus Panel (2004) NIH Consensus Statement on total knee replacement December 8-10, 2003. J Bone Joint Surg Am 86: 1328-35.

37. Ravi B, Croxford R, Austin PC, Lipscombe L, Bierman AS, et al. (2013) The relation between total joint arthroplasty and risk for serious cardiovascular events in patients with moderate-severe osteoarthritis: propensity score matched landmark analysis. BMJ 347: f6187. doi: 10.1136/bmj.f6187. 